\title{
A Formal Validation Approach for Holonic Control System Specifications
}

\author{
Paulo Leitão \\ Polytechnic Institute of Bragança \\ Quinta $S^{t a}$ Apolónia, Apartado 134 \\ 5301-857 Bragança, Portugal \\ Email: pleiao@ipb.pt
}

\author{
Armando W. Colombo \\ Schneider Electric GmbH \\ Steinheimer Str. 117, \\ 63500 Seligenstadt, Germany \\ Email: armando.colombo@modicon.com
}

\author{
Francisco Restivo \\ Faculty of Engineering-University of Porto \\ Rua Dr. Roberto Frias, \\ P-4200-465 Porto, Portugal \\ Email: fjr@fe.up.pt
}

\begin{abstract}
The holonic manufacturing paradigm allows a new approach to the emergent requirements faced by the manufacturing world, through the concepts of modularity, decentralisation, autonomy, re-use of control software components. The formal modelling and validation of the structural and behavioural specifications of holonic control systems assumes a critical role. This paper discusses the formal validation of the Petri Net models designed to represent the behaviour and specifications of the holon classes defined at ADACOR architecture.
\end{abstract}

\section{INTRODUCTION}

Nowadays the globalisation, the emergent technologies and the customer demands require the flexible operation in a global scale and lead to the need for agile, flexible and adaptive manufacturing control approaches.

To face these complex requirements, new distributed manufacturing control paradigms were proposed, presenting distributed structures based in autonomous and cooperative entities that have the ability to respond promptly and correctly to external changes, differing from conventional approaches due to their inherent capability to adapt to changes without external interventions.

The holonic manufacturing systems (HMS) [1] is only one paradigm for the next generation of manufacturing and processing technologies, amongst others, like fractal [2] and bionic [3] manufacturing systems. In spite of their different origins, these theories present similar concepts and characteristics, suggesting the idea that manufacturing systems will continue to need a hierarchical structure besides the increased autonomy assigned to individual entities.

In recent years, several architectures and developments in holonic manufacturing were reported, covering different application domains, such as manufacturing scheduling and control, materials handling, machine controllers and assembly systems, such as referred in [1], [4]-[7] and others compiled in [8].

One of these holonic architectures, proposed during the last two years, is the ADACOR architecture [9], which defines a set of autonomous, self-organised and intelligent holons aiming to improve the performance of control system in industrial stochastic scenarios, characterised by the frequent occurrence of unexpected disturbances.
The formal modelling and validation of the structural and behavioural specifications of the ADACOR-holons and the interaction of these holons aiming to reach the manufacturing control functions assumes a critical aspect. The validation phase is crucial to guarantee the correctness of designed model, that is, that the model represents correctly the specifications of the real system. The validation phase of the development methodology proposed by the authors is based on the mathematical background (functional analysis and linear algebra) associated with a kind of Petri Net (PN) model tailored for holonic control system modelling. Additionally, it allows simulating the designed model, verifying the behaviour of modelled system over the time.

The paper presents the formal validation of structural and behavioural specifications of the ADACOR-holon classes presented in [10]. This formal validation is mainly based on the qualitative and quantitative analysis of the Petri net models addressed in [10], which are supported by the software package PASCELL [11], [12].

The paper is organised as follows: Section 2 makes an overview of ADACOR architecture, describing its objective and focus, the holon classes and the interaction between them to achieve manufacturing control functions, and Section 3 describes the issues related to the edition of the PN-models using an appropriated software tool. Section 4 presents the qualitative analysis of PN model for the task holon class that belongs to the ADACOR architecture and Section 5 presents the performance analysis for the same PN-model. At last, Section 6 summarises the conclusions.

\section{ADACOR HOLONS BEHAVIOUR}

The ADACOR architecture proposes a holonic approach to face the dynamic and agile adaptation to disturbances in flexible manufacturing systems [9]].

\section{A. Overview of ADACOR Architecture}

ADACOR, based in the holonic manufacturing paradigm, is built upon a set of autonomous and cooperative holons, where each holon represents a manufacturing entity, such as a numerical control machine, a robot, and a manufacturing order. The implementation of the holons entities is done using the agent technology, taking advantage of modularity, 
decentralization, and dynamic and complex structures design features.

According the generalization concept of the object-oriented paradigm, the ADACOR architecture groups the manufacturing holons into product, task, operational and supervisor holon classes [13].

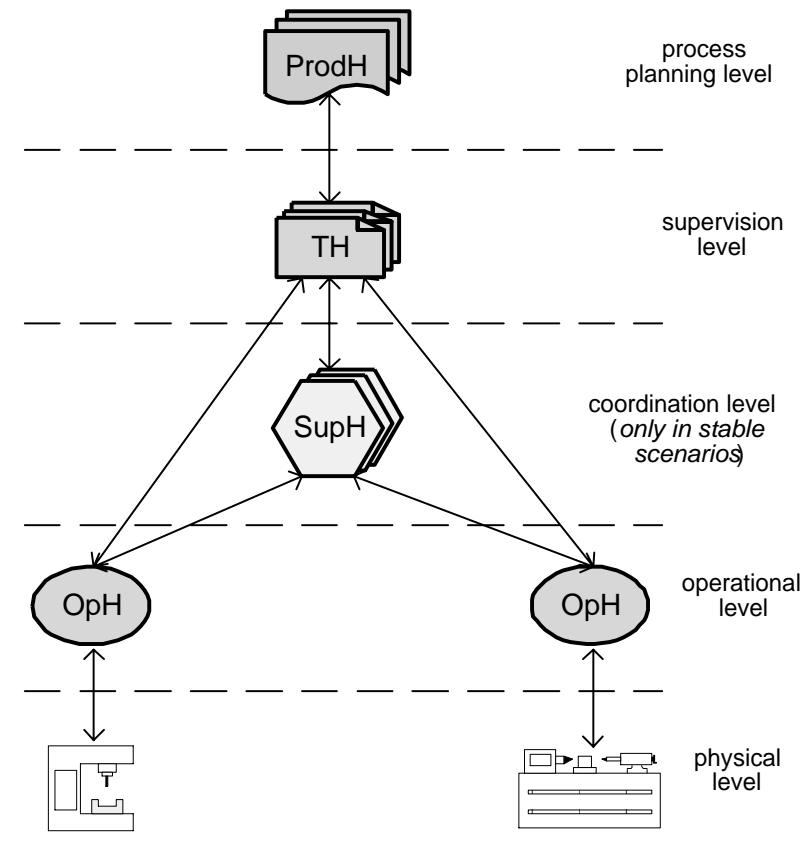

Fig. 1. Interdependency between ADACOR Holon Classes

Each product is represented by a product holon that contains all knowledge related to the product and process. Manufacturing orders to be executed in the factory plant are represented by task holons, which are responsible for the control and supervision of their execution. The operational holons represent the manufacturing resources, such as operators and robots, managing its behaviour and agenda according to the resource goals, constraints and skills.

The product, task and operational holons are quite similar to the product, order and resource holons, presented at the PROSA reference architecture [1]. The supervision holon, presents different characteristics from the staff holons defined in PROSA, introducing coordination and global optimisation in decentralised control approaches, coordinating several operational and supervisor holons. In normal operation, the supervisor holon supervises and regulates the activity of the holons under its domain, while when a disturbance occurs, these holons may have to find their way without the help of the supervisor holon. The supervisor holon is also responsible for the group formation and their dynamic evolution according to the environment context, based in pre-defined clusters of holons, combining synergies, aggregating skills and offering the combined services to external entities in the manufacturing system. These groups can be formed to build a shop floor, a manufacturing cell, or a machine equipped with a set of tools, assuming the supervisor holon the role to coordinate each group.

Besides the autonomy and cooperation capabilities, the holons present in ADACOR architecture, present learning and self-organisation capabilities [14]. The learning capability allows that the holon increases its performance and behaviour according to the actual environment and experience, supporting the decision-making process. The self-organisation aims the dynamic adaptation of the system to react to unexpected disturbances, facing the customer demands, economical trends or machine failures. The degree of efficiency of selforganisation capability is dependent from how the learning mechanisms are implemented.

\section{B. Formal Specification of Holons' Behaviour}

The manufacturing control applications are normally complex software, which is difficult to understand and to design, even using multi-agent or holonic systems. In order to simplify this complexity, it is often used modelling tools that intent to understand and synthesise the system behaviour. In complex systems, as manufacturing control systems are, is crucial the development and validation of models that represent the dynamic and static behaviour of the system, appearing the modelisation aggregated to the design and the implementation phases, aiming to simplify the development of manufacturing control systems.

PN is a formal modelling tool, graphical and mathematical, well suitable to model and analyse the structure and functional aspects of complex event-driven systems, characterized as being concurrent, asynchronous, parallel, stochastic and with high distribution degree, such as flexible manufacturing systems. More details about PN theory and mathematical fundamentals are out of the scope of this work. We recommend the readers to consult the following references [11], [12], [15][17].

In [10] it is used a kind of Petri net [11], tailored for production management and control modelling purposes, as a formal methodology to model the behaviour of the ADACORholon classes in a bottom-up approach. The individual model of each ADACOR-Holon class uses special temporised transitions to model activities execution, which can be exploded into a more detailed and refined level. These sub-models, according the degree of refinement, are the different software control modules of the hardware, i.e., a formal representation of the holons.

In distributed systems and more particularly in the control of distributed systems, after designing PN models, in this case for the ADACOR holon classes, the following qualitative specifications must be validated: boundedness of resources, reversibility, deadlock-freeness, conservativeness of resources, mutual exclusion relationships, among others.

The PN-models without time parameter associated to their elements have a very strong mathematical background (functional analysis and linear algebra), that allows verifying the qualitative specifications.

The quantitative analysis of the PN-models with the parameter time associated to some of their elements allows to 
perform the formal validation of performance specifications, i.e., performance indexes associated with the modelled Holons can be generated and used for optimisation purposes.

\section{EDITION OF HOLON PN-MODELS}

The edition, simulation and validation will be done using the PASCELL software tool [11], [12]. This software tool, developed in $\mathrm{C}++$ programming language, allows the edition, simulation, and analysis of generalised and temporised Petri Net models. The qualitative analysis is based on discrete-event simulation and structural analysis of the matrix representation of the net. The quantitative analysis is performed by means of the simulation of the temporized Petri net models. Details about the used temporised models are out of the scope of this work. For more information, the reader should consult the references [11], [12].

Fig. 2 to 5 illustrate the edition of the PN-model for all ADACOR Holon classes, detailed described in [10], which will support the future qualitative and quantitative analysis, and the subsequent formal validation of specifications.

Fig. 2 represents the edition of the PN model for the ADACOR product holon. Basically, the product holon is waiting for new product orders; each production order will generate a new thread to handle its execution. In each thread, initially it is elaborated several alternative plans for the execution of the product, and it is verified if the required parts or raw material are available on the storage system.

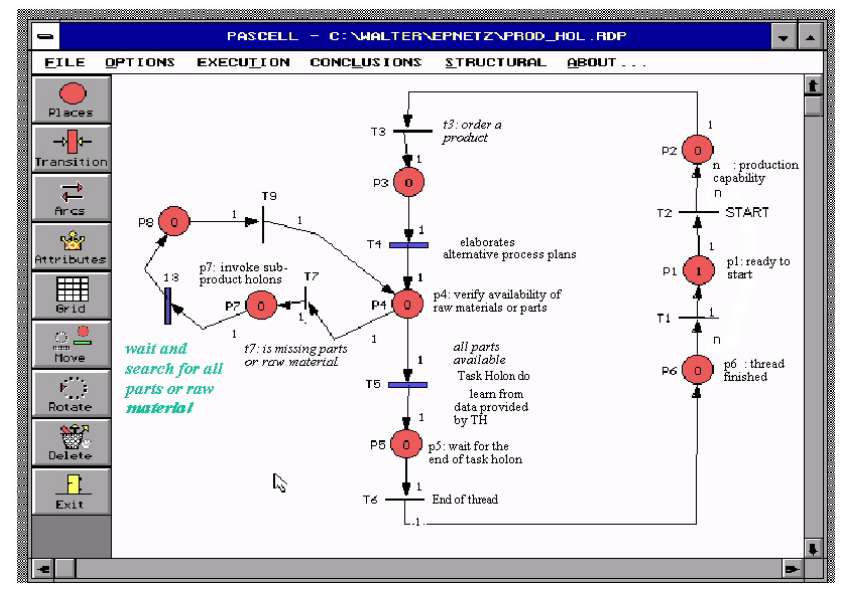

Fig. 2. Model of the Product Holon

If the raw materials or parts are not available, the product holon interact with other product holons to request the execution of the sub-products. When all sub-products or raw materials are available, it launches a task holon that will supervise the manufacturing order execution.

After launching a new task holon, the thread will wait for the conclusion of the manufacturing order. At this moment, it receives the relevant information concerning the execution of the part, allowing to learn from the current manufacturing order execution, in order to elaborate more efficient and accurate process plans in the future.

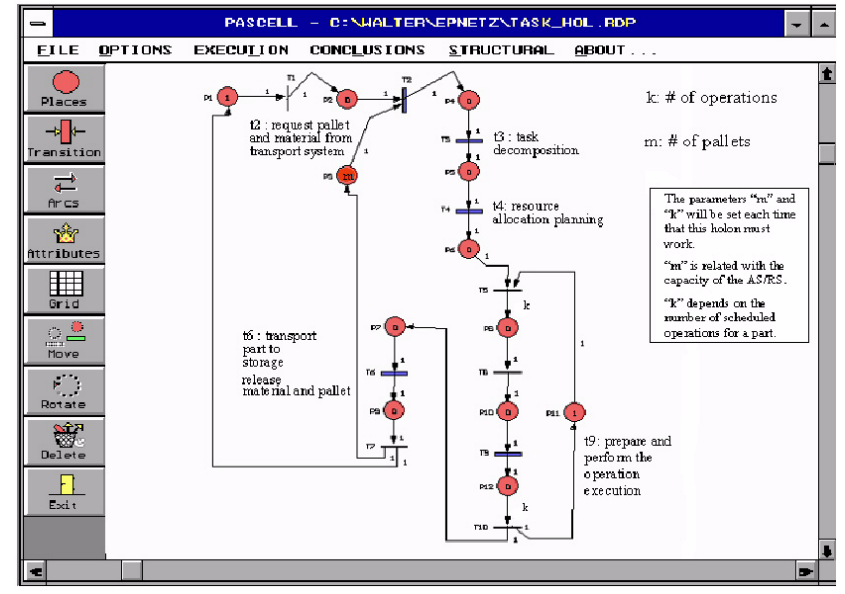

Fig. 3. Model of the Task Holon

The task holon behaviour model, edited in Fig.3, comprises mainly the following activities: request a pallet and material to the storage system, allocation of the operations belonging to the manufacturing order to the available resources, execution of the resource allocation plan, and release of the pallet.

The resource allocation plan comprises the preparation of the operation execution, which involves the transportation of the parts to the machine where the parts will be processed, and the execution of set-ups in the machine, if necessary, and the monitoring of the operation execution.

The operational holon behaviour, which model is edited in Fig.4, acts as a reactive server, in the sense that it is waiting for new operations (proposed by the supervisor holon or by the task holons) and it has the possibility to execute monitoring, scheduling and disturbances handling.

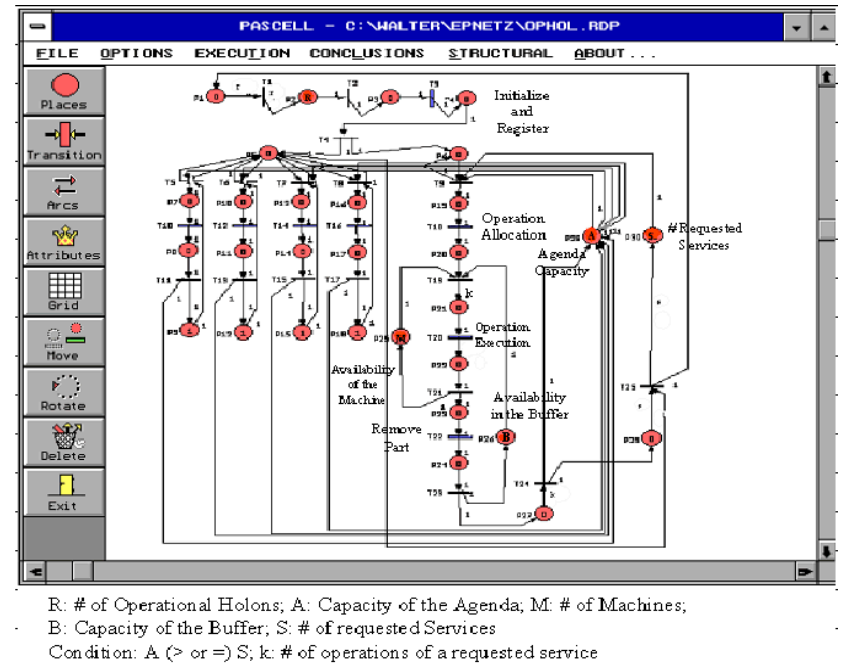

Fig. 4. Model of the Operational Holon

The sub-behaviours are handled asynchronously using threads, so that the execution of one process doesn't block the execution of another process; for example, when monitoring 
the execution of an operation, the operational holon can handle the announcement of new operations or execute scheduling. The monitoring, disturbance handling and scheduling activities are performed concurrently.

The behaviour model of the supervisor holon, edited in Fig. 5, acts simultaneously as a server and a client: as a server waiting for requests and as a client generating optimised schedules that are sent to the operational holons.

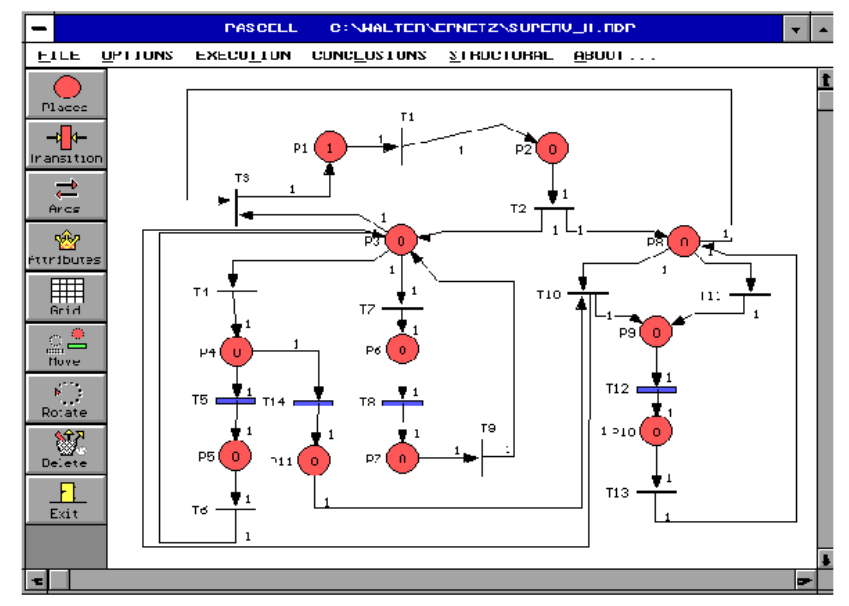

Fig. 5. Model of the Supervisor Holon

The decision-making activity is related to the actions associated to monitoring, scheduling and disturbance handling, presenting more complexity than to the one presented at operational holons due to the need to handle with aggregated lower holons knowledge and skills. As the supervisor holon co-ordinates several operational and/or supervisor holons, it manages the group of holons under its coordination domain, aggregating the skills and capacity of the operational holons, when they join to the group.

The transitions of ADACOR Holon models use a deterministic distribution associated to the time unit to trigger. The arcs in the PN-models have associate weights: some have the unitary weight and others have the weight associated to the number of instances presented in the system, such as the number of products, resources, pallets, and operations.

\section{QUalitative AnALYSis}

After the edition of the PN-models, the next phase is related to the structural analysis of the PN-models and the validation of specifications.

\section{A. Basic Concepts}

The qualitative analysis, based on the structural analysis of the models, allows verifying the structural and behavioural properties of the model, extracting conclusions about the functionality of the system, such as the existence of deadlocks, the bounded capacity of resources, and the existence of structural and behavioural conflicts in the system [18]. For the purposes of this work, the following properties will be examined [17]:
- Boundedness: A PN is $\mathbf{k}$-bounded if each place in the net gets at most $\mathbf{k}$ tokens for all markings in the reachability set. This guarantees that the number of resources (number of tokens at the places, which can be number of machines, places in the buffers, etc) is limited. If the model is kbounded and $\mathbf{k}=1$, then the PN is safe.

- Reversibility: A PN is reversible if the initial marking is reachable from all reachable markings, guaranteeing that the model can reinitialise from itself.

- Liveness: A PN is live if for any marking in the reachability set it is possible to fire any transition in the net. This property is very important since the liveness guarantees the absence of deadlocks. In other words, a reachable marking $\mathbf{m}$ is a deadlock if no transition is enabled in $\mathbf{m}$, which allow to change to another reachable marking. A $\mathrm{PN}$ is deadlock free if no reachable marking is a deadlock.

- Conservativeness: A PN is conservative if it is not created or destroyed new tokens in the net. This guarantees that for example it is not destroyed parts (material under processing aiming the final product) from the system.

The available methods for the analysis of behavioural properties are the following: reachability tree and linear algebra method [17].

The first method is a graph built upon the reachability tree of the PN, and shows the evolution of the tokens over the net model. The nodes of the tree represent a marking in the net that corresponds to a state of the system, and the arcs represents the transitions that can occur from each node. The building of reachability tree is done from the definition of an initial marking, $\mathbf{m}_{0}$, which implies that this method is dependent on the initial marking of the PN. For that marking it is analysed which transitions lead to a new marking $\mathrm{m}$. If this marking still not exist, it is attributed a name to it, $\mathbf{m}_{k}$, different from the existing ones. For each new marking in the tree it is repeated the process done for $\mathbf{m}_{0}$. In case of a marking, which no transition trigger a new marking, the situation is designated by deadlock.

From the analysis of the reachability tree it is possible to verify the behavioural properties, such as the existence of deadlocks and the boundedness. However, for complex PNs the construction of the reachability tree explodes, making impracticable the structural analysis using this method.

The second method is based in mathematical linear algebra background using the incidence matrix. The incidence matrix, $\mathbf{W}$, represents the PN structure, describing how the nodes in the PN are interconnected, and in this way describing the evolution of the tokens by the several places, according the trigger of a sequence of transitions. Formally, the incidence matrix can be defined as a $(n \times m)$ matrix of integers,

$$
W=\left[a_{i j}\right]
$$

where $\mathbf{a}_{i j}$ represents the number of marks removed from the input places (negative integers), or the number of marks stored in the output places (positive integers). 
The method is based in the following equation,

$$
m_{k}=m_{0}+W \cdot T
$$

which allows to determine a new marking in the model $\left(\mathrm{m}_{k}\right)$ from the knowledge of the incidence matrix, the initial marking $\left(\mathrm{m}_{0}\right)$ and a sequence of triggered transitions $(\mathrm{T})$. In order to support the analysis of the behavioural properties it is defined the concepts of P-invariants and T-invariants, which uses the incidence matrix.

The P-invariant is a $(n \times 1)$ nonnegative integer vector $\mathbf{x}$ satisfying:

$$
x^{T} W=0
$$

Considering $\mathrm{r}=\operatorname{rank}(\mathrm{W})$, there are (n-r) minimal $\mathrm{P}$ invariants and a PN is covered by $\mathrm{P}$-invariants if all its places belong to some P-invariants. A siphon is a set of places such that every transition that outputs to one of these places also inputs from one of these places. Thus, if all the places in a siphon have no tokens, then the corresponding set of places will never have a token.

Similarity, a T-invariant is a $(m \times 1)$ nonnegative integer vector $\mathbf{y}$ satisfying:

$$
W y=0
$$

Using the P-invariants it is possible to verify the liveness and boundedness of a PN: a PN is live and bounded if it is covered by $\mathrm{P}$-invariants, all the P-invariants are marked with tokens, and none of the siphons is ever cleared of tokens. Additionally, using the P-invariants and T-invariants it is possible to determine the properties of the PN [11], [17]:

- A PN is conservative if there exists an $(n \times 1)$ vector $\mathbf{x}$ such that $x^{T} W=0$;

- A PN is repetitive if there exists a $(m \times 1)$ vector $\mathbf{y}$ such that $W y \geq 0$;

- A PN is not reversible if $W y=0$ admits only the trivial solution. Even a nontrivial solution only guarantees partial reversibility.

Like the reachability tree method, the analysis of PN using linear algebra methods over the incidence matrix has its disadvantages, such as the solution for the Eq. 2 is only a necessary condition to verify the reachability, and the determination of the liveness requires a hard search for the solution.

In this work, the behavioural properties are analysed using linear algebra methods, provided by the PASCELL software tool.

\section{B. Results of Qualitative Analysis}

In this paper the qualitative analysis will be exemplified and illustrated by means of only one holon, namely the Task Holon due to the easy understand that it provides.

Fig. 6 illustrates the Task Holon as a Petri net, but by means of an algebraic form. This matrix, that is the incidence matrix $\mathrm{W}$ referred in the Eq. 1, represents the holon structure in another form than a graphic. As example, the firing of

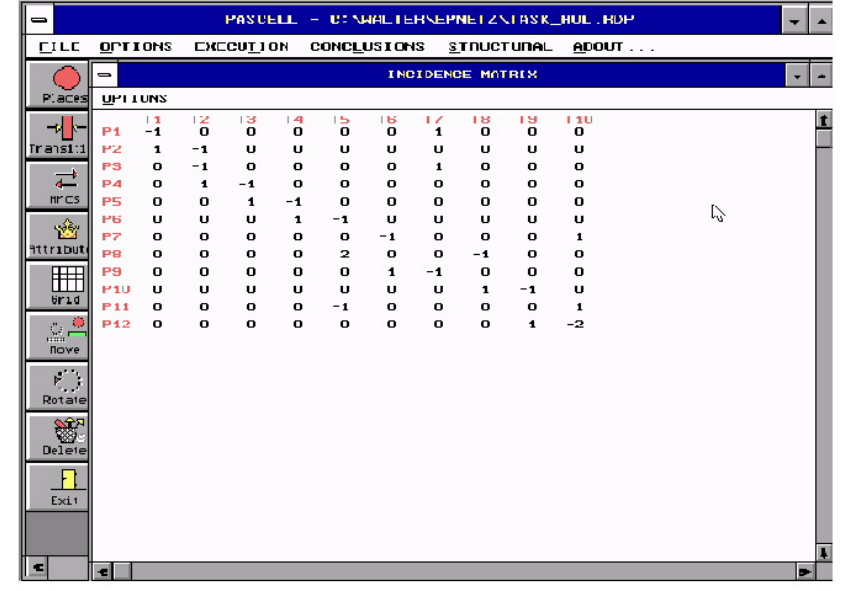

Fig. 6. Incidence Matrix for the Task Holon

transition $t_{1}$ will remove a token from place $P_{1}$ and put a token in the place $\mathrm{P}_{2}$.

Additionally, it was considered that a manufacturing order includes two operations, which will be scheduled for transition $t_{9}$.

The results of the structural analysis of the incidence matrix are illustrated in Fig. 7. This structural analysis shows that the PN-model is live (satisfying the necessary condition), which guarantees the deadlock freeness, i.e. the execution of a manufacturing order is done without to be blocked in a determined intermediate state.

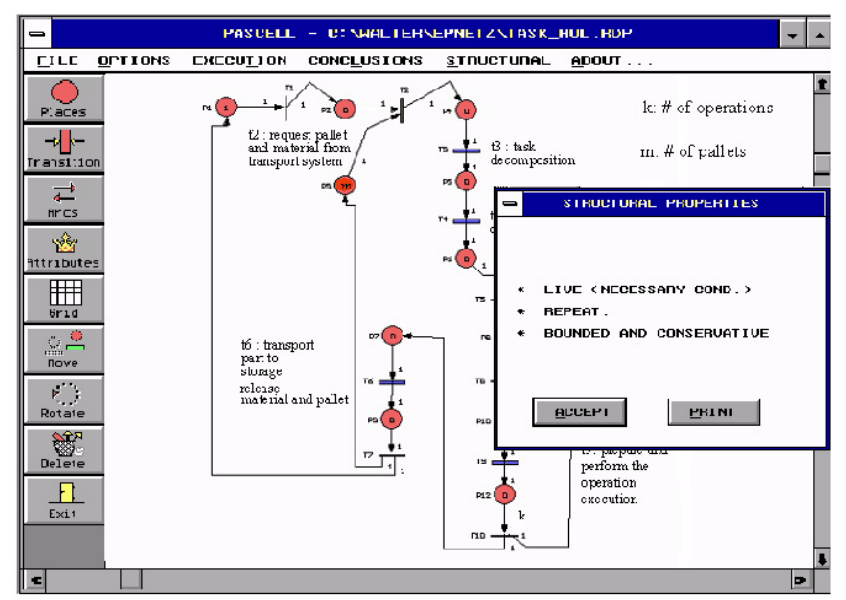

Fig. 7. Structural Analysis of the Task Holon Model

The PN-model is repetitive, which means that the PN returns to the initial state (marking), existing well defined work cycles in the execution of manufacturing orders.

The PN-model is conservative, which means that the pallets does not disappear neither are created new pallets during the execution of the operations associated to the manufacturing order. Additionally, the model is bounded, which means that the number of manufacturing orders in the system is limited to the maximum value of $\mathbf{m}$, due to the existence of the monitor 
place that regulates the available pallets, and the number of operations that belongs to each manufacturing order is also limited to the value $\mathbf{k}$ (in this case equal to two).

Other characteristics of the PN-model for the Task Holon can be found from the analysis of this incidence matrix. Fig. 8 presents the set of P-supports of the incidence matrix. This set is related to the p-invariants referenced in Eq. 3. A brief view about their structures allows confirming mutual exclusion relationships among places and of course, functions and resources involved in the Holon-structure and behaviour.

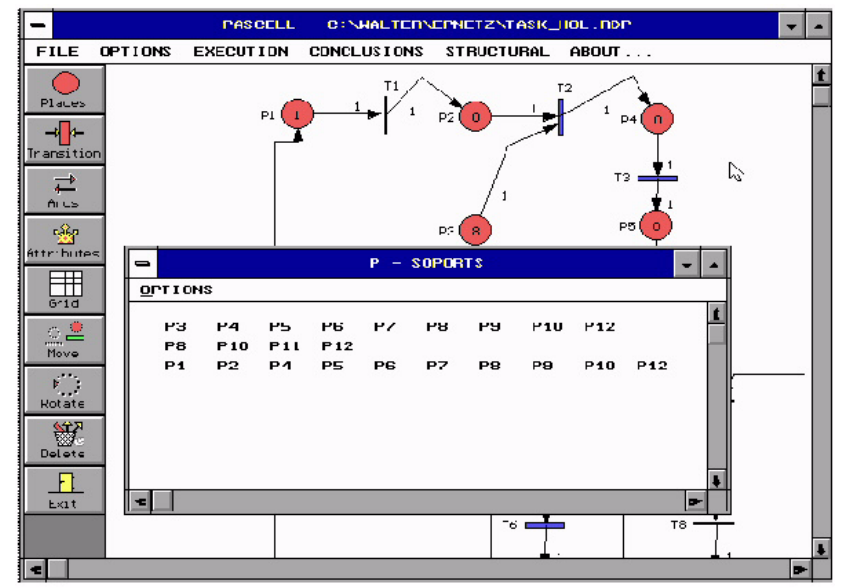

Fig. 8. P-invariants for the Incidence Matrix of the Task-Holon Model

At this point a very exact qualitative analysis has been performed, without simulation and only by means of linear algebra and functional analysis.

Only if the model presents all necessary properties, like those addressed above, it is possible to be sure that the model is correct from the functional analysis viewpoint, and it can be seen as a virtual representation of the Holon. In this case, the next step is to use the same models, but now with the time parameter associated to the elements of the model, to perform a quantitative analysis of the holon's behaviour.

\section{Quantitative Analysis}

The quantitative analysis, also designated by performance analysis, requires the introduction of time parameter associated to the transitions. The editor draws these transitions with color blue and they are representing time-consuming activities. The rest of the transitions are drawn with black color.

As in the qualitative analysis, only the quantitative analysis for the task holon will be described in the paper, taking into consideration the temporisation of the Holon-models presented in Table I.

Related to the task holon model, the transitions $t_{2}$ to $t_{4}$ are computational activities, namely, the pallet request, the task decomposition, and the resource allocation planning, being estimated 1 second per transition.

The transition $t_{6}$ represents the activity related to the preparation of the operation execution, which involves the transportation of the part to the machine where the part will
TABLE I

Time AsSOCIATED to THE TRANSITIONS

\begin{tabular}{|c||c|c|}
\hline Holon model & Transition & Time Units \\
\hline \hline Product & $\mathrm{T}_{4}$ & 1 \\
& $\mathrm{~T}_{5}$ & 80 \\
& $\mathrm{~T}_{8}$ & 90 \\
\hline Task & $\mathrm{T}_{2}$ & 1 \\
& $\mathrm{~T}_{3}$ & 1 \\
& $\mathrm{~T}_{4}$ & 1 \\
& $\mathrm{~T}_{6}$ & 10 \\
& $\mathrm{~T}_{9}$ & 20 \\
\hline Operational & $\mathrm{T}_{2}$ & 1 \\
& $\mathrm{~T}_{8}$ & 1 \\
& $\mathrm{~T}_{9}$ & 1 \\
& $\mathrm{~T}_{10}$ & 2 \\
& $\mathrm{~T}_{11}$ & 1 \\
& $\mathrm{~T}_{17}$ & 1 \\
& $\mathrm{~T}_{21}$ & 20 \\
& $\mathrm{~T}_{23}$ & 10 \\
\hline Supervisor & $\mathrm{T}_{4}$ & 1 \\
& $\mathrm{~T}_{5}$ & 1 \\
& $\mathrm{~T}_{7}$ & 1 \\
& $\mathrm{~T}_{9}$ & 2 \\
& $\mathrm{~T}_{13}$ & 5 \\
\hline
\end{tabular}

be processed, and the execution of set-ups in the machine, if necessary. For this study it is considered that this activity takes 10 seconds.

The transition $t_{9}$ includes the transportation of the part to the storage system and releases the pallet, after the execution of all operations. Also includes the transfer of relevant information about the product execution to the product holon. The time associated to this transition is dependent of the actual capacity of the transport system, but for the study is was estimated 20 seconds.

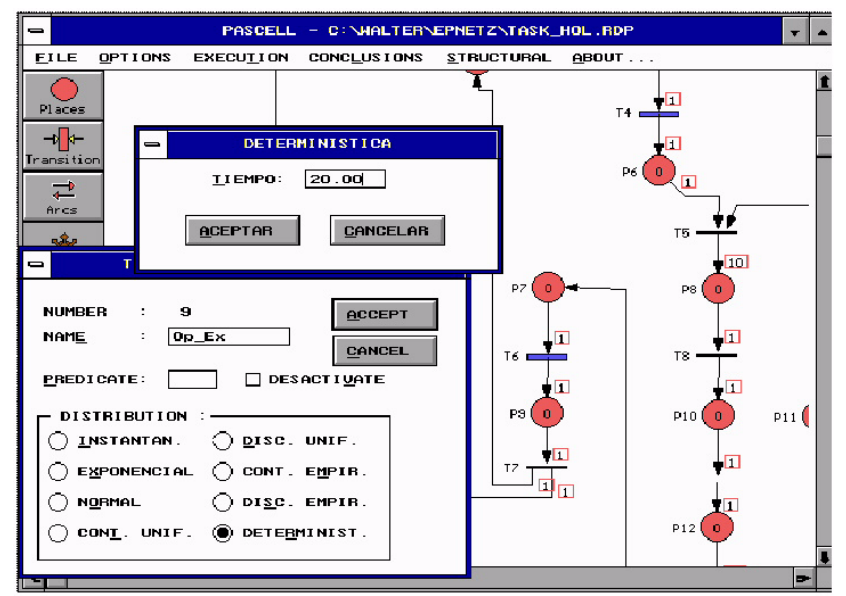

Fig. 9. Assignment of Time Parameter to the Transitions

A screen shot with the time assignment to the transitions is 
illustrated in Fig. 9. In this case, transition $t_{9}$ has associated a deterministic time parameter with value 20 [time-units], according to Table I.

During the net evolution, black transitions are transitions with atomic-firing. Blue transitions have a three-phases firing process. In the first phase, the transition is marking enabled and captures the marks of the pre-condition-places (without time). In a second phase, the transition is evolving, i.e. the modelled activity is being performed. This phase has associated a certain number of time-units as parameter, those corresponding to the modelled activity. As soon as this time elapses, the transition starts the third phase and put the corresponding marks in the post-condition-places.

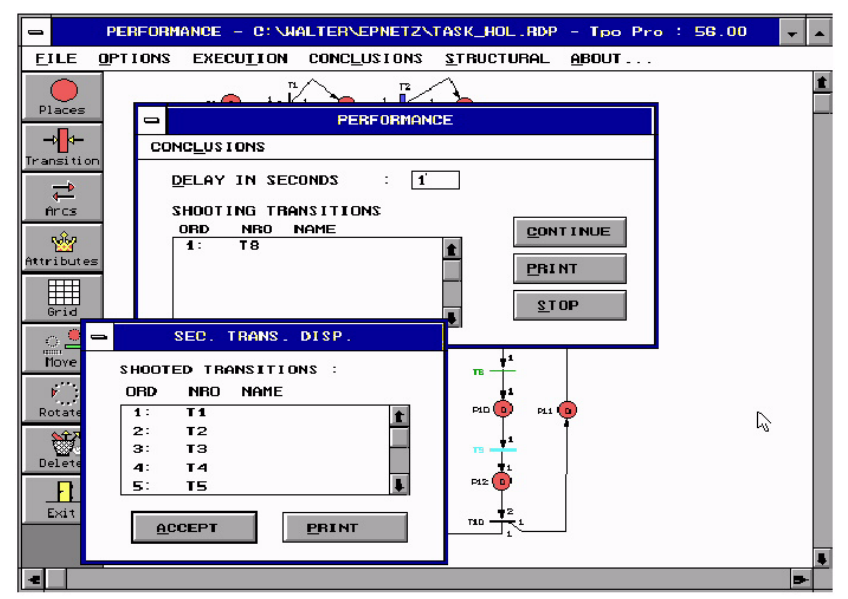

Fig. 10. Evolution of the Firing Process

This evolution is shown in Fig. 10. A green marked transition is firing (atomic-firing) at this time and is evolving within its third phase. A heavenly marked transition is evolving within its second phase. Thus, Fig. 10 shows that after 56 [time-units] of evolution, the transition $t_{8}$ is firing and the transition $t_{9}$ is evolving.

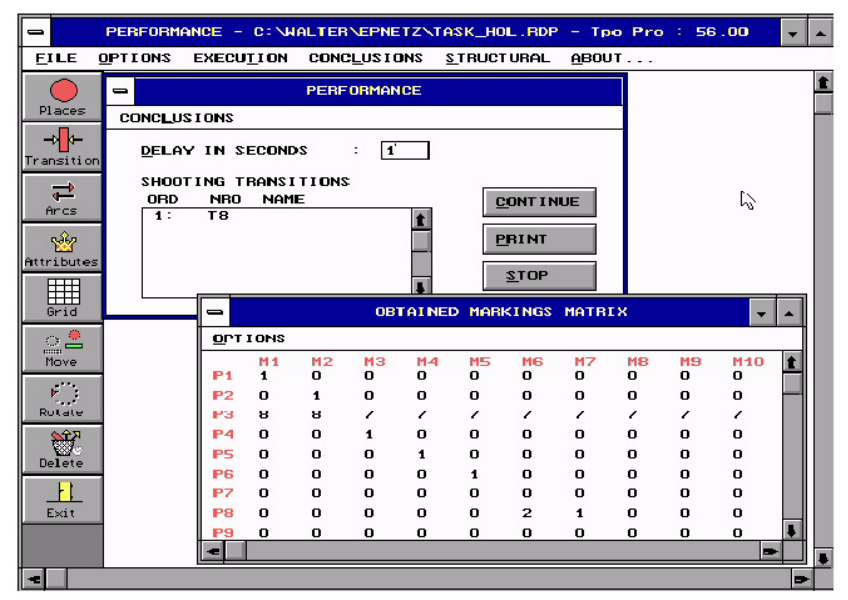

Fig. 11. States Reached after 56 Time Units

The current coverability/reachability graph, i.e. the states reached by the model after 56 [time-units] is illustrated in
Fig. 11. This screen shot, together with Fig. 12, allows to perform a very precise analysis of the evolution of the holon behaviour, answering to pertinent questions, such as which states have been reached, which activities or functions have been performed and with which frequency (in comparison to the others) and which is the history of the evolution of the holon.

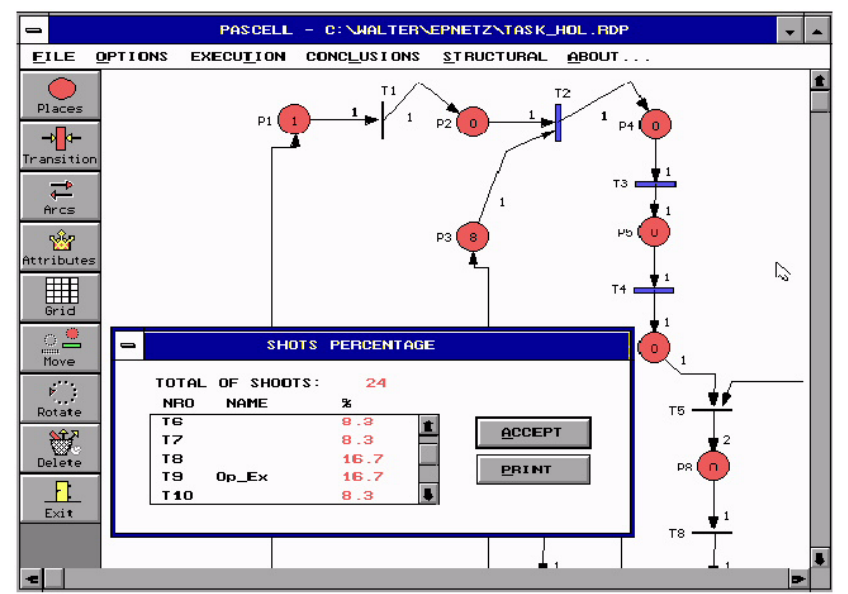

Fig. 12. Percentage of Transition Shoots after 56 Time Units

All the above addressed information about the timed evolution of the HOLON can be summarized with a Gantt diagram, which is shown in Fig. 13 and reflect the temporal sequence of system functioning. The time assignment of Table I to the Task Holon can be verified in the diagram of Fig. 13. Moreover, cyclic evolution, existence of bottlenecks, mutual exclusion activities, etc., can be easily discovered and of course, optimisation strategies can be then proposed and online verified.

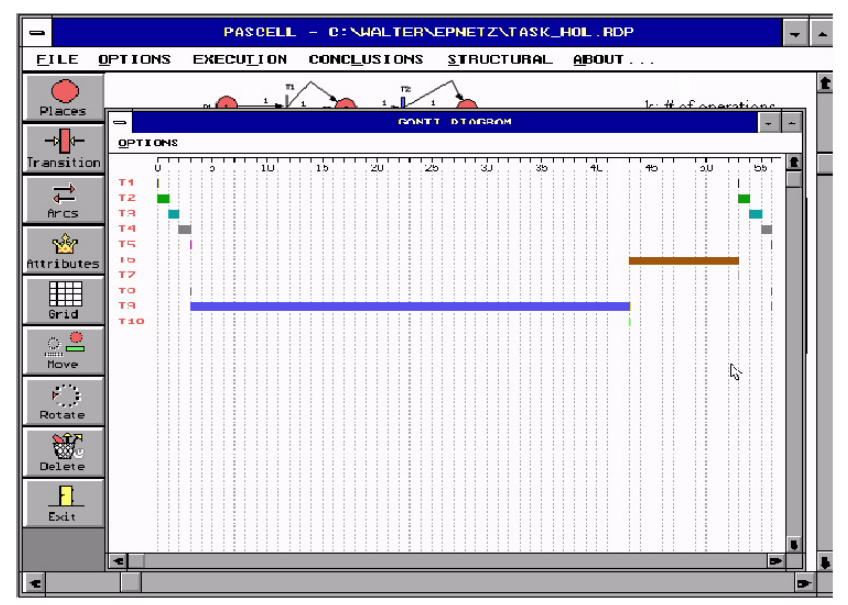

Fig. 13. Gantt Diagram for the Performance Analysis

The procedure for qualitative and quantitative analysis, described previously for the task holon, has been repeated for the others ADACOR holon PN-models, allowing for the validation of all specifications (structural and behavioural) 
presented in [9], [10], [13], [14].

\section{CONClusions And Future Work}

The holonic paradigm has been applied to solve the emergent requirements faced by the manufacturing systems, such as the demand for agile, distributed and flexible manufacturing control systems.

The formal modelisation and validation of the structural and behavioural specifications of holonic control systems assumes a critical aspect, with little attention devoted to it in the research community.

This paper describes the formal validation of holonic control systems using a kind of Petri Net, tailored for production management and control modelling purposes. The structural and behaviour specifications of the Holon classes defined at ADACOR architecture have been presented in [10] and now have been formally validated.

Future efforts will be oriented to the application of Objectoriented and Colored Petri net models for modelling and formal validation of complex Holonic control systems, since the models used here are unable to represent this kind of manufacturing control structures, characterized by a big number of different components (Holons).

\section{ACKNOWLEDGMENTS}

The first author would like to acknowledge the PRODEP program for the financial support granted (PRODEP III$5.3 / 2000)$.

\section{REFERENCES}

[1] H. Van Brussel, J. Wyns, P. Valckenaers, L. Bongaerts and P. Peeters, "Reference Architecture for Holonic Manufacturing Systems: PROSA", Computers In Industry, vol. 37, 1998, pp. 255-274.

[2] H.J. Warneke, The Fractal Company, Springer-Verlag, 1993.

[3] N. Okino, "Bionic Manufacturing System", CIRP, Flexible Manufacturing Systems Past-Present-Future, J. Peklenik (ed.), 1993, pp. 73-95.

[4] K. Fisher, "Agent-Based Design of Holonic Manufacturing Systems", Journal of Robotics and Autonomous Systems, Elsevier Science B.V., vol.27, pp 3-13

[5] M. Fletcher, V. Marík and P. Vrba, "Design Issues in Holonic Inventory Management and Material Handling Systems", in Knowledge and Technology Integration in Production and Services: Balancing Knowledge and Technology in Production and Service Life Cycle, V. Marik, L. M. Camarinha-Matos and H. Afsarmaneh (eds.), Kluwer Academic Publisers, 2002, pp. 271-280.
[6] L. Gou, P. B. Luh and Y. Kyoya, "Holonic manufacturing scheduling: architecture, cooperation mechanism, and implementation", Computers in Industry, vol. 37, 1998, pp. 213-231.

[7] A. W. Colombo, R. Neubert and R. Schoop, " A Solution to Holonic Control Systems", in Proceedings of the $8^{\text {th }}$ IEEE International Conference on Emerging Technologies and Factory Automation, Sophia/Nice, France, 2001, pp 489-498.

[8] Journal of Applied Systems Studies, Special Issue on Industrial Applications of Multi-Agent and Holonic Systems, 2(1), 2001.

[9] P. Leitão and F. Restivo, "Adaptive Production Control Systems", in Proceedings of special session on Agent-based Intelligent Automation and Holonic Control Systems of the 28th Annual Conference of the IEEE Industrial Electronics Society, Sevilla, Spain, 5-8 November, 2002, pp 2968-2973.

[10] P. Leitão, A.W. Colombo and F. Restivo, "An Approach for the Formal Specification of Holonic Control Systems", to appear in the $1^{\text {st }}$ International Conference on Applications of Holonic and Multi-Agent Systems (HoloMAS), September 1-3, 2003, Prague, Republic Czech.

[11] A.W. Colombo, Development and Implementation of Hierarchical Control Structures of Flexible Production Systems Using High-Level Petri Nets, in "Manufacturing Automation Series", Klaus Feldmann (Ed.) / PhD-Thesis, 216 pages, MeisenbachVerlag Bamberg, 1998.

[12] A.W. Colombo, R. Carelli and B. Kuchen, "A Temporized Petri Net Approach for Designing, Modelling and Analysis of Flexible Production Systems", The International Journal of Advanced Manufacturing Technology, 13 (3), 1997, Springer Verlag, pp. 214-226.

[13] P. Leitão and F. Restivo, "Identification of ADACOR Holons for Manufacturing Control", in Proceedings of $7^{\text {th }}$ IFAC Workshop on Intelligent Manufacturing Systems, Budapest, Hungary, 6-8 April, 2003, pp 109114.

[14] P. Leitão and F. Restivo, "Towards Autonomy, Self-Organisation and Learning in Holonic Manufacturing", Multi-Agent Systems and Applications III, $3^{\text {rd }}$ International/Central and Eastern European Conference on Multi-Agent Systems, V. Marik, J. Mller and M. Pechoucek (eds), Lecture Notes in Artificial Intelligence, vol. 2691, Springer-Verlag, 2003, pp 544-553.

[15] T. Murata, "Petri Nets: Properties, Analysis and Applications", Proceedings of the IEEE, 77(4), 1989, pp 541-580.

[16] A.W. Colombo and R. Carelli, "Petri Nets for Designing Manufacturing Systems", Computer-Assisted Management and Control of Manufacturing Systems, cap. 11, S.G. Tzafestas (ed.), 1997, Springer-Verlag.

[17] A. Desrochers and R. Al-Jaar, Applications of Petri Nets in Manufacturing Systems-Modeling, Control and Performance Analysis, 1995, IEEE Press.

[18] K. Feldmann,C. Schnur and A.W. Colombo, "Modularised, Distributed Real-Time Control of Flexible Production Cells, Using Petri Nets", Control Engineering Practice, International Journal of IFAC, pp. 10671078. August 1996 